Unfallchirurg 2011 114:380-381

DOI 10.1007/s00113-011-1965-2

(c) Springer-Verlag 2011

D.W. Sommerfeldt ${ }^{1} \cdot$ H.-G. Dietz ${ }^{2}$

${ }^{1}$ Abteilung für Kinder- und Jugendtraumatologie, Altonaer Kinderkrankenhaus gGmbH, Hamburg

${ }^{2}$ Kinderchirurgische Klinik im Dr. von Haunerschen Kinderspital, Klinikum der Universität, LMU München

\title{
Kinder- und Jugendtraumatologie - untere Extremität
}

deutung ist. In diesem Zusammenhang ist auch die hier referierte Zusammenfassung der Multicenterstudie zu den Epiphyseolysen der distalen Tibia zu sehen. Nur durch solche Sammelstudien mit großen Fallzahlen und ausreichend langen Nachbeobachtungszeiten können unsere Erkenntnisse gesichert werden und ein ausreichender wissenschaftlicher Evidenzgrad erreicht werden.

Wie in der April-Ausgabe auch haben wir Wert darauf gelegt, Themen aus der Kinder- und Jugendtraumatologie zu wählen, bei denen es durchaus noch Diskussionsbedarf gibt:

- Ab welchem Alter behandelt man die Femurschaftfraktur operativ?

- Sind operative Eingriffe nach Patellaluxation am wachsenden Skelett obsolet?

- Haben Plattenosteosynthesen an der proximalen Tibia im Kindesalter ausgedient?

- Gibt es CRPS überhaupt bei Kindern?

Dies sind nur 4 von vielen Fragen, die nach Lektüre der Beiträge hoffentlich beantwortet werden können. Eben so viele Fragen werden sich erst beim Lesen aufdrängen, um dann vielleicht in Form von Leserbriefen gestellt zu werden.

Wir wollen nochmals ausdrücklich betonen, dass gerade die Kinder- und Jugendtraumatologie mit ihren z. T. geringen Fallzahlen zu den einzelnen Verletzungen ein Tätigkeitsfeld ist, in dem der Austausch von Erfahrungen und das Sammeln von Erkenntnissen von größter Be-

\section{In der Kinder- und Jugendtraumatologie sind der Austausch von Erfahrungen und das Sammeln von Erkenntnissen von größter Bedeutung}

Passend dazu möchte ich es als Leiter der Sektion Kindertraumatologie der Deutschen Gesellschaft für Unfallchirurgie nicht versäumen, Sie alle herzlich zu unserer Jahrestagung in Freiburg am 17. und 18. Juni 2011 einzuladen (http://www. skt2011.de/).

Eine weitere Möglichkeit in den wissenschaftlichen Diskurs auf dem Gebiet der Kinder- und Jugendtraumatologie einzutreten bietet sich dann vom 25. - 28.10.2011 beim Jahreskongress der DKOU in Berlin. Auch hier ist unser Fachgebiet wieder mit zahlreichen Veranstaltungen vom Kurs für den Berufsanfänger bis zur "Tipps-und-Tricks-Sitzung“ für den erfahrenen Kinderunfallchirurgen vertreten.
Und wem das alles nicht vollständig genug ist, dem darf ich zu guter Letzt noch ein Lehrbuch empfehlen, dass fast zeitgleich mit diesen beiden Ausgaben zur Kinder- und Jugendtraumatologie erscheint:

„Praxis der Kinder- und Jugendtraumatologie" fasst auf 552 Seiten mit 1200 Abbildungen, davon 300 in Farbe, den derzeitigen Wissensstand zu unserem spannenden Fachgebiet umfassend und trotzdem praxisrelevant zusammen (http://www.springer.com/medicine/ book/978-3-642-12934-6).

Viel Spaß bei der Lektüre wünschen Ihnen

Für die Sektion Kindertraumatologie der DGU e.V.

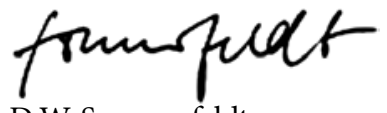

D.W. Sommerfeldt

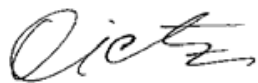

H.-G. Dietz

\section{Korrespondenzadresse}

PD Dr. D.W. Sommerfeldt

Abteilung für Kinder- und Jugendtraumatologie,

Altonaer Kinderkrankenhaus g GmbH Bleickenallee 38, 22763 Hamburg dirk.sommerfeldt@kinderkrankenhaus.net 
Hier steht eine Anzeige.

黛 Springer 